\title{
TGF $\beta 1$ exacerbates blood-brain barrier permeability in a mouse model of hepatic encephalopathy via upregulation of MMP9 and downregulation of claudin-5
}

\author{
Matthew A McMillin', Gabriel A Frampton ${ }^{1}$, Andrew P Seiwell ${ }^{1}$, Nisha S Patel', Amber N Jacobs² and \\ Sharon DeMorrow ${ }^{1,3,4}$
}

Recent studies have found that vasogenic brain edema is present during hepatic encephalopathy following acute liver failure and is dependent on increased matrix metalloproteinase 9 (MMP9) activity and downregulation of tight junction proteins. Furthermore, circulating transforming growth factor $\beta 1$ (TGF $\beta 1$ ) is increased following liver damage and may promote endothelial cell permeability. This study aimed to assess whether increased circulating TGF $\beta 1$ drives changes in tight junction protein expression and MMP9 activity following acute liver failure. Blood-brain barrier permeability was assessed in azoxymethane (AOM)-treated mice at 6,12 , and $18 \mathrm{~h}$ post-injection via Evan's blue extravasation. Monolayers of immortalized mouse brain endothelial cells (bEnd.3) were treated with recombinant TGF $\beta 1$ ( $\mathrm{TTGF} \beta 1$ ) and permeability to fluorescein isothiocyanate-dextran (FITC-dextran), MMP9 and claudin-5 expression was assessed. Antagonism of TGF $\beta 1$ signaling was performed in vivo to determine its role in blood-brain barrier permeability. Blood-brain barrier permeability was increased in mice at $18 \mathrm{~h}$ following AOM injection. Treatment of bEnd.3 cells with rTGF $\beta 1$ led to a dose-dependent increase of MMP9 expression as well as a suppression of claudin- 5 expression. These effects of rTGF $\beta 1$ on MMP9 and claudin-5 expression could be reversed following treatment with a SMAD3 inhibitor. AOM-treated mice injected with neutralizing antibodies against TGF $\beta$ demonstrated significantly reduced blood-brain barrier permeability. Blood-brain barrier permeability is induced in AOM mice via a mechanism involving the TGF $\beta 1$-driven SMAD3-dependent upregulation of MMP9 expression and decrease of claudin-5 expression. Therefore, treatment modalities aimed at reducing TGF $\beta 1$ levels or SMAD3 activity may be beneficial in promoting blood-brain barrier integrity following liver failure.

Laboratory Investigation (2015) 95, 903-913; doi:10.1038/labinvest.2015.70; published online 1 June 2015

Acute liver failure (ALF) can lead to many detrimental effects outside the liver, including a systemic inflammatory response, increased energy expenditure and catabolism, and multi-organ failure. $^{1-3}$ However, one of the most difficult to treat complications of ALF arises from the development of neurological deficits, called hepatic encephalopathy (HE). HE has classically been identified as a reduction of the liver's ability to metabolize neurotoxins, such as ammonia, which accumulate in the brain generating neurological impairment. ${ }^{4}$ Associated with cerebral ammonia accumulation is cytotoxic brain edema and the development of Alzheimer's Type II astrocytes in the basal ganglia of HE patients. ${ }^{5}$ However, for neurotoxic metabolites to enter the brain, the blood-brain barrier (BBB), which is not permeable to these neurotoxins in normal physiological conditions, must be disrupted. ${ }^{6}$
Microvascular endothelial cells that line the vasculature of the BBB are different from other endothelial cells as they lack fenestrations, have more extensive tight junctions, and have reduced pinocytic vesicular transport. ${ }^{7}$ Tight junctions, which are functional barriers created by joining together endothelial cells, are made up of cytoplasmic accessory proteins (zona occludens-1, -2, and -3), which anchor the actin cytoskeleton to transmembrane proteins (claudins and occludin). ${ }^{8}$ Although direct dysregulation of tight junctions can cause vasogenic edema, matrix metalloproteinases (MMPs) have been demonstrated to digest tight junction proteins, allowing for multiple levels of BBB dysregulation. ${ }^{9}$ During ALF, decreased zona occludens- 2 protein expression has been shown to precede BBB permeability. ${ }^{10}$ Furthermore, it has been shown that claudin-5 and occludin are decreased in mice

\footnotetext{
${ }^{1}$ Department of Internal Medicine, Texas A\&M Health Science Center College of Medicine, Temple, TX, USA; ${ }^{2}$ University of Texas Health Science Center, Houston, TX, USA ${ }^{3}$ Baylor Scott \& White Health Digestive Disease Research Center, Temple, TX, USA and ${ }^{4}$ Central Texas Veterans Health Care System, Temple, TX, USA

Correspondence: MA McMillin, PhD, Department of Internal Medicine, Texas A\&M Health Science Center, Central Texas Veterans Health Care System, Building 205, 1901S 1st St Temple, TX 76504, USA

E-mail: mcmillin@medicine.tamhsc.edu
}

Received 25 November 2014; revised 9 April 2015; accepted 26 April 2015 
with HE. ${ }^{11}$ MMP9 upregulation has also been identified to induce $\mathrm{BBB}$ permeability during the later stages of HE. ${ }^{12}$ However, the specific signaling pathways influencing BBB permeability during $\mathrm{HE}$ are not well classified and warrant investigation.

Transforming growth factor beta 1 (TGF $\beta 1$ ) is a signaling protein involved in many processes including immune system modulation, cell proliferation, cell differentiation, and apoptosis. ${ }^{13,14}$ During HE it has been shown that TGF $\beta 1$ is found in the circulation of rats with hepatic failure. ${ }^{15}$ Furthermore, we have found TGF $\beta 1$ present in the serum of mice following toxic liver injury. ${ }^{16}$ In regards to $\mathrm{BBB}$ permeability, evidence exists that TGF $\beta 1$ can directly affect endothelial cell permeability. Endothelial lung cells grown on monolayers treated with TGF $\beta 1$ demonstrated significantly increased permeability following treatment. ${ }^{17}$ Also, retinal endothelial cells treated with $\operatorname{TGF} \beta 1$ were found to increase MMP9 expression, which increased permeability of these endothelial cells. ${ }^{18}$

The hypothesis of this study is that the BBB is disrupted during $\mathrm{HE}$ and that circulating $\operatorname{TGF} \beta 1$ contributes to increased vascular permeability via the upregulation of MMP9 and disruption of tight junction proteins. These combined mechanisms would allow a greater degree of toxin entry into the brain and exacerbate the pathogenesis of HE.

\section{MATERIALS AND METHODS Materials}

Immortalized mouse brain endothelial cells (bEnd. 3 cells) were purchased from American Type Culture Collection (Manassas, VA). The 24-well transwell inserts were purchased from Corning (Tewksbury, MA). Antibodies against MMP9 were purchased from Santa Cruz Biotechnology (Santa Cruz, CA). Antibodies against claudin-5 were purchased from Invitrogen (Grand Island, NY). Antibodies against albumin used for immunocytochemistry were purchased from Genetex (Irvine, CA). Antibodies against albumin for tissue immunohistochemistry were bought from Bethyl Laboratories (Montgomery, TX). Neutralizing antibodies for TGF $\beta$ (antiTGF $\beta$ ) and recombinant TGF $\beta 1$ protein ( $\mathrm{rTGF} \beta 1$ ) were purchased from R\&D systems (Minneapolis, MN). SMI71 antibodies were purchased from Covance (Princeton, NJ). All quantitative PCR primers were purchased from SABiosciences (Frederick, MD). The TGF $\beta$ receptor II (TGF $\beta$ RII) antagonist, GW788388, was purchased from Tocris Bioscience (Minneapolis, MN). All other chemicals were purchased from Sigma-Aldrich (St. Louis, MO) unless otherwise noted, and were of the highest grade available.

\section{Experimental Animals and Hepatic Encephalopathy Model}

Mouse in vivo experiments were performed using male C57Bl/6 mice (25-30 g; Charles River Laboratories, Wilmington, MA). Mice were allowed free access to drinking water and standard mouse chow and were housed in constant temperature, humidity, and $12 \mathrm{~h}$ light-dark cycling. Cages were assigned to random groups and mice received either a single intraperitoneal injection of $100 \mathrm{mg} / \mathrm{kg} \mathrm{AOM}$ to induce ALF and HE or an equal amount of saline for control animals. After injection, mice were placed on heating pads set to $37^{\circ} \mathrm{C}$ and under heating lamps to ensure they maintained normal body temperature. Further, mice were supplied with hydrogel and rodent chow on their cage floor to ensure they had access to food and hydration. After the first $12 \mathrm{~h}$, and every subsequent $4 \mathrm{~h}$, mice were injected subcutaneously with $500 \mu \mathrm{l}$ of a $5 \%$ dextrose solution to prevent hypoglycemia. Mice were removed from the study if they underwent a 20\% weight loss. Neurological and behavioral assessments of the mice were performed as previously described. ${ }^{16,19}$

To suppress the activity of circulating $\mathrm{TGF} \beta, \mathrm{TGF} \beta$ neutralizing antibodies ( $R \& D$ Systems, Minneapolis, MN) were administered via a single intraperitoneal injection at $1 \mathrm{mg} /$ $\mathrm{kg} 2 \mathrm{~h}$ before AOM injection. This treatment has previously been shown to delay the onset of neurological symptoms after AOM injection. ${ }^{16}$ For this study, no differences were detected between mice treated with $\mathrm{AOM}$ alone and mice treated with $\mathrm{AOM}$ and immunoglobulin G1 (unpublished observations); therefore, mice treated with $\mathrm{AOM}$ alone were appropriate controls for mice co-treated with AOM and TGF $\beta$-neutralizing antibodies. Mice in all groups were killed at $18 \mathrm{~h}$ following AOM injection. All experiments performed complied with the Scott \& White Memorial Hospital IACUC regulations on animal experiments (protocol \#2012-019-R).

\section{In vivo BBB Permeability Measurements}

To assess in vivo permeabilization of the $\mathrm{BBB}$ in the $\mathrm{AOM}$ mouse model of HE, a modified Evan's blue dye assay was performed in vehicle and $\mathrm{AOM}$ mice. ${ }^{20,21}$ Mice were anesthetized with isoflurane inhalation and an incision was made in the neck to expose the carotid artery. Evan's blue dye was injected $(5 \mathrm{mg} / \mathrm{ml} ; 500 \mu \mathrm{l})$ and allowed to circulate for 20 min at which time mice were killed. Killed mice were then perfused transcardially with $50 \mathrm{ml}$ of cold phosphate buffered saline (PBS), the meninges were removed, and the brain was blotted dry. The brain stem and cerebellum were removed, and the two remaining hemispheres were homogenized with $1.5 \mathrm{ml}$ ice-cold trichloroacetic acid $(50 \% \mathrm{v} / \mathrm{v})$ in a glass homogenizer. The resulting homogenates were centrifuged for $10 \mathrm{~min}$ at $10,000 \mathrm{~g}$, and absorbance of the supernatant was read at $620 \mathrm{~nm}$. In vivo permeabilization was measured using the same methods in mice treated with neutralizing antibodies against $\operatorname{TGF} \beta$ as well.

\section{In vitro Permeability Assessments}

To assess endothelial cell permeabilization in vitro, monolayers of bEnd. 3 cells were seeded at a density of $5.0 \times 10^{4}$ cells $/ \mathrm{cm}^{2}$ onto 24 -well Transwell inserts with a $0.4-\mu \mathrm{m}$ pore. After cells grew into a confluent monolayer $(48-72 \mathrm{~h}$ ), cells were treated with AOM $(100 \mathrm{ng} / \mathrm{ml}$ to $10 \mu \mathrm{g} / \mathrm{ml}), \operatorname{rTGF} \beta 1$ $(0.5 \mathrm{ng} / \mathrm{ml}$ to $5.0 \mathrm{ng} / \mathrm{ml})$, GW788388 $(1 \mu \mathrm{M})$, specific 
inhibitor of SMAD3 (SIS3) $(1 \mu \mathrm{M})$, or dimethyl sulfoxide (DMSO) for $24 \mathrm{~h}$. Following treatment, inserts and chambers were washed with PBS and media was replaced with phenolred free media. $10 \mathrm{kDa}$ FITC-dextran $(10 \mathrm{mg} / \mathrm{ml} ; 10 \mu \mathrm{l})$ was added to the upper wells for $1 \mathrm{~h}$. Fluorescence (excitation $494 \mathrm{~nm}$; emission $520 \mathrm{~nm}$ ) was read in the upper and lower chambers, and the permeability coefficient was determined using the following formula: $:^{22} P_{\text {dextran }}=\left(R F U^{\text {lower }} / R F U^{\text {upper }}\right)$ $(V)(1 / t)(1 / A)$, where $R F U$ is the relative fluorescent units in the upper and lower wells, $V$ is the volume of the bottom well, $t$ is the time that the FITC-dextran was allowed to diffuse, and $A$ is the total surface area of the monolayer $\left(\mathrm{cm}^{2}\right)$. Permeability coefficients were normalized by setting basal cell monolayers to a value of 1 to minimize variability between trials.

\section{Immunofluorescence and Immunohistochemistry}

For brain immunohistochemistry, free-floating $30-\mu \mathrm{m}$ sections were sectioned and put into 12-well plates containing PBS. Sections were put in $0.5 \%$ hydrogen peroxide to quench endogenous peroxidase activity. Brain sections were blocked in $5 \%$ goat serum before overnight incubation of specific antibodies against albumin. ${ }^{21}$ Secondary antibodies and DAB peroxidase substrate were supplied from Vector Labs (Burlingame, CA). Incubations and staining development were performed according to the manufacturer's protocols. The sections were viewed using an Olympus BX40 microscope with an Olympus DP25 imaging system (Olympus, Center Valley, PA).

Free-floating immunofluorescence of the brain was performed on $30-\mu \mathrm{m}$ sections. Brains were initially blocked in $5 \%$ goat serum before overnight incubation with specific antibodies against albumin and SMI71. Immunocytochemistry in bEnd. 3 cells was performed using the same methods with antibodies against MMP9 and claudin-5. Immunoreactivity was visualized using Dylight 488- or Cy3-conjugated secondary antibodies and counterstained with 4',6-diamidino-2-phenylindole (DAPI). Slides were viewed and imaged using a Leica TCS SP5-X inverted confocal microscope (Leica Microsystems, Buffalo Grove, IL).

\section{Quantitative PCR}

RNA was extracted from bEnd.3 cells using the RNeasy mini kit from Qiagen (Valencia, CA) as per manufacturer's protocols. RNA content of isolated samples was calculated using a Thermo Scientific Nanodrop 2000 (Rockford, IL). An iScript cDNA synthesis kit (Bio-Rad, Hercules, CA) was used to amplify $1 \mu \mathrm{g}$ of RNA per reaction in a MyCycler thermal cycler (Bio-Rad). cDNA was loaded onto 96-well plates with iTaq universal SYBR green supermix (Bio-Rad) along with commercially available primers designed against mouse claudin-5, MMP9, and glyceraldehyde 3-phosphate dehydrogenase (GAPDH). Quantitative PCR (qPCR) was performed using a Strategene Mx3005P qPCR system (Santa Clara, CA), and a $\Delta \Delta \mathrm{CT}$ analysis was performed using basal bEnd. 3 cells as controls. ${ }^{23,24}$ Data for all experiments are expressed as mean relative mRNA levels \pm s.e.m. $(n=4)$.

\section{Immunoblotting}

Homogenization of bEnd.3 cells was accomplished by scraping cells in lysis buffer supplemented with $1 \%$ protease inhibitor cocktail. Protein content in cell lysates from bEnd.3 cells was quantitated using a BCA protein assay (Thermo Scientific, Rockford, IL). SDS-PAGE gels were loaded with 10-20 $\mu \mathrm{g}$ of protein diluted in Laemmli buffer per each tissue sample. Specific antibodies against claudin-5, MMP9, and $\beta$ actin were used. All imaging was performed on an Odyssey 9120 Infrared Imaging System (LI-COR, Lincoln, NE). Data are expressed as fold change in fluorescent band intensity of target antibody divided by the loading control, $\beta$-actin. The values of basal bEnd. 3 cells were used as a baseline and set to a relative protein expression value of 1 . All treatment groups were expressed as changes of fluorescent band intensity of target antibody to $\beta$-actin relative to basal. All band intensity quantifications were performed using ImageJ software (National Institutes of Health, Bethesda, MD). Data for all experiments are expressed as mean relative protein \pm s.e.m. $(n=4)$.

\section{Statistical Analysis}

All statistical analyses were performed using Graphpad Prism software (Graphpad Software, La Jolla, CA). Results were expressed as mean \pm s.e.m.. For data that passed normality tests, the Student's $t$-test was used when differences between two groups were analyzed, and analysis of variance was used when differences between three or more groups were compared followed by the appropriate post hoc test. If tests for normality failed, two groups were compared with a Mann-Whitney $U$-test. When tests for normality failed with more than two groups, a Kruskal-Wallis ranked analysis was used. Differences were considered significant when the $P$-value was $<0.05$.

\section{RESULTS \\ The BBB is Disrupted Following AOM-Induced Liver Failure}

C57Bl/6 mice were treated with the hepatotoxin AOM and Evan's blue extravasation was assessed after 6, 12, or $18 \mathrm{~h}$. Mice that were treated for $18 \mathrm{~h}$ with AOM had significantly increased Evan's blue dye present in their brains compared with those perfused with dye alone (Figure 1a). Interestingly, mice that underwent Evan's blue extravasation assays at 6 and $12 \mathrm{~h}$ post AOM injection had essentially no change in Evan's blue dye penetrance into their brain compared with untreated mice. As Evan's blue binds albumin, this provides evidence that the BBB is being disrupted to a large enough degree to allow the passage of large proteins into the brain following ALF. Representative pictures of untreated, $0-12 \mathrm{~h}$, and $18 \mathrm{~h}$ brains support the findings from our absorbance measures (Figure 1b). Albumin immunofluorescence was performed in 


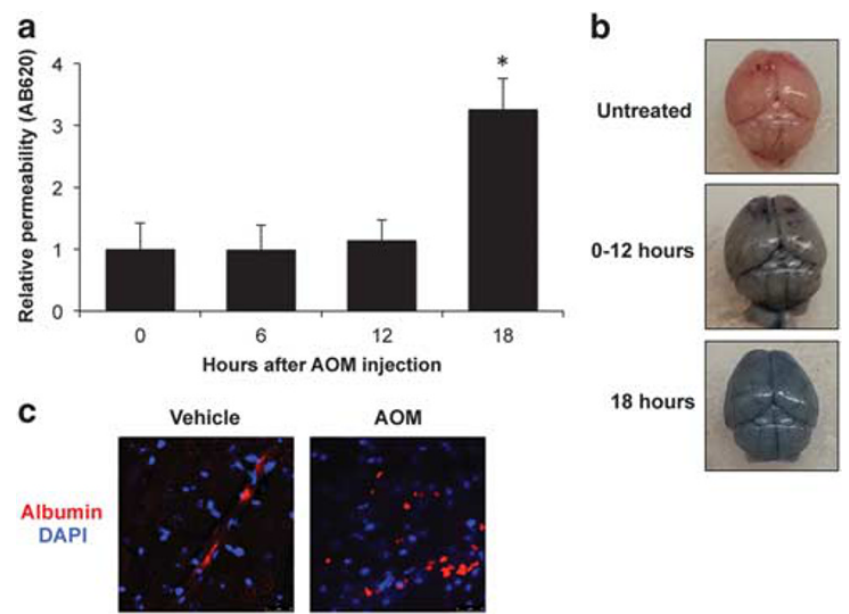

Figure 1 The BBB is disrupted in the later stages of HE. (a) Evan's blue dye permeability assay of AOM mice at indicated time points following AOM injection $(n=4)$. Permeability was measured by measuring absorbance of Evan's blue dye $(620 \mathrm{nM})$. (b) Representative pictures of brains from mice that had no Evan's blue dye extravasation (untreated), mice infused with Evan's blue dye after AOM treatment from 0-12 h, and mice infused with Evan's blue dye $18 \mathrm{~h}$ after AOM injection. (c) Immunofluorescence in vehicle and AOM cortex for albumin (red) and DAPI (blue). Data in the permeability assay are reported as mean \pm s.e.m. ${ }^{*}=P<0.05$ compared with $0 \mathrm{~h}$ mice.

the cortex of vehicle and AOM mice. This demonstrated that in vehicle mice there is only slight residual albumin staining in cerebral microvessels, while in AOM-treated mice albumin immunoreactivity is found diffusely throughout the tissue (Figure 1c). Together, these findings demonstrate that BBB permeability is increased in mice treated with $\mathrm{AOM}$ as is indicated by the presence of both Evan's blue dye and positive albumin immunofluorescence in the cortex during later stages of AOM-induced HE.

\section{Circulating TGF $\beta 1$ can Disrupt the BBB}

Initially, the effects of AOM treatment on endothelial cell permeability were assessed using confluent bEnd. 3 monolayers in transwell chambers as an in vitro model of the BBB. Treatment of bEnd. 3 monolayers with $100 \mathrm{ng} / \mathrm{ml}$ to $10 \mu \mathrm{g} / \mathrm{ml}$ of AOM did not increase monolayer permeability as assessed by diffusion of $10 \mathrm{kDa}$ FITC-dextran across the transwell (Figure 2a). Therefore, it appears that some other circulating factor that is released during AOM-induced hepatotoxicity contributes to increased BBB permeability observed following AOM treatment. To determine whether TGF $\beta 1$ could induce permeability, monolayers were treated with increasing doses of rTGF $\beta 1$ and permeability was assessed. At doses of 1.0 and $5.0 \mathrm{ng} / \mathrm{ml}$ of $\mathrm{rTGF} \beta 1$, which are physiologically relevant levels in mice, ${ }^{25}$ there were significant increases in BBB permeability (Figure $2 \mathrm{~b}$ ). To ensure that the resulting increased permeabilization was entirely due to TGF $\beta 1$ signal transduction, monolayers were treated with $\operatorname{rTGF} \beta 1$ in combination with a TGF $\beta$ RII antagonist, GW788388. The previously seen increased permeability of $1.0 \mathrm{ng} / \mathrm{ml}$ rTGF $\beta 1$-treated bEnd. 3 monolayers
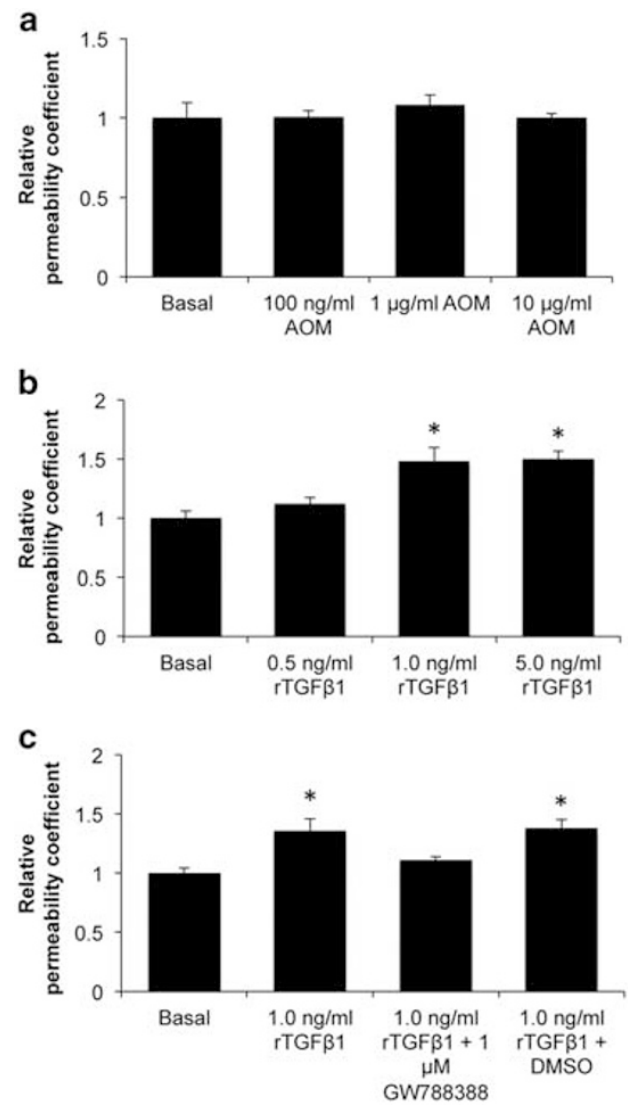

Figure 2 Monolayers of brain endothelial cells are permeabilized by treatment with rTGF $\beta 1$. (a) Transwell chambers seeded with a monolayer of bEnd.3 cells were treated with indicated concentrations of AOM for $24 \mathrm{~h}$. Diffusion of 10-kDa FITC-dextran from the top to bottom chamber and subsequent measurement of fluorescence was employed to assess monolayer permeability. (b) Monolayers of bEnd.3 cells plated on transwells were treated with indicated doses of rTGF $\beta 1$ for $24 \mathrm{~h}$. Permeability was assessed by 10-kDa FITC-dextran diffusion from the top chamber to the bottom chamber and subsequent measurement of fluorescence (excitation 494/emission 520). (c) Monolayers of brain endothelial cells were treated with $\operatorname{rTGF} \beta 1$, the TGF $\beta$ RII antagonist GW788388, or DMSO (vehicle for GW788388) for $24 \mathrm{~h}$. Permeability was assessed by measuring diffusion of 10-kDa FITC-dextran from the top chamber to the bottom chamber via fluorescence measurement (excitation 494/emission 520). Data in the monolayer assays are reported as mean \pm s.e.m. ${ }^{*}=P<0.05$ compared with basal bEnd. 3 cells.

was significantly reduced following treatment with $1 \mu \mathrm{M}$ GW788388 (Figure 2c). This demonstrates that TGF $\beta 1$ receptor-mediated signaling is responsible for the effects of TGF $\beta 1$ on inducing brain endothelial cell monolayer permeability.

\section{MMP9 is Upregulated in Endothelial Cells by TGF $\beta 1$}

To determine whether TGF $\beta 1$-induced increase in brain endothelial cell permeability was due to an increase of MMP9 activity, bEnd. 3 cells were treated with $\operatorname{rTGF} \beta 1$ and MMP9 expression assayed. Treatment with $\operatorname{rTGF} \beta 1$ led to a dosedependent increase of MMP9 mRNA expression, with treatments of $0.5 \mathrm{ng} / \mathrm{ml} \mathrm{rTGF} \beta 1$ and higher generating a 
significant increase (Figure 3a). To determine whether this treatment led to increased protein levels of MMP9, immunofluorescence was performed and a dose-dependent increase in MMP9 immunostaining was observed (Figure 3b). Quantification of MMP9 immunofluorescence determined that doses of $1.0 \mathrm{ng} / \mathrm{ml}$ and $5.0 \mathrm{ng} / \mathrm{ml}$ of $\operatorname{rTGF} \beta 1$ led to a significant increase in immunoreactivity (Figure 3c). These data demonstrate that TGF $\beta 1$ may generate its effects on permeability through upregulation of MMP9.

\section{Claudin- 5 is Downregulated by TGF $\beta 1$ in bEnd. 3 Cells}

As TGF $\beta 1$ was shown to increase permeability of bEnd. 3 monolayers and induce an upregulation of MMP9, further investigation into tight junction protein regulation was warranted. The tight junction proteins claudin-5, occludin, zona occludens-1, and zona occludens- 2 were assessed following $\operatorname{rTGF} \beta 1$ treatment. There were no significant changes in occludin, zona occludens-1, and zona occludens- 2 expression when assessed by western blotting, qPCR, or immunofluorescence (data not shown). However, there were changes observed in claudin-5. Treatment of bEnd. 3 cells with rTGF $\beta 1$ led to a significant suppression of claudin-5 mRNA expression (Figure 4a). This effect translated into a reduction of claudin-5 protein with significant suppression at doses of $0.5 \mathrm{ng} / \mathrm{ml} \mathrm{rTGF} \beta 1$ and greater (Figure $4 \mathrm{~b}$ ). To determine whether these changes in gene and protein expression led to a functional disruption of the tight junction, immunofluorescence against claudin- 5 was performed, demonstrating staining localized to the cell membrane of basal cells. However, when bEnd. 3 cells were treated with $\operatorname{rTGF} \beta 1$, claudin- 5 immunostaining became increasingly cytoplasmic as doses increased, indicating a disruption of tight junctions (Figure 4c). These data demonstrate that TGF $\beta 1$ is able to downregulate claudin-5 and disrupt its localization to tight junctions in brain endothelial cells.

\section{Modulations of MMP9 and Claudin-5 via TGF $\beta 1$ are Dependent on SMAD3}

SMAD3 is one of the intracellular proteins that transduce extracellular signaling of TGF $\beta 1$ to the nucleus, thereby generating effects on transcription. ${ }^{26}$ To determine whether TGF $\beta 1$ is exerting its effects through a SMAD3-dependent mechanism, bEnd. 3 cells were treated with $\operatorname{rTGF} \beta 1$ and the SMAD3 antagonist SIS3. Treatment of monolayers with TGF $\beta 1$ and SIS 3 was able to alleviate the permeability caused due to TGF $\beta 1$ treatment alone (Figure $5 \mathrm{a}$ ).

Treatment of bEnd. 3 cells with TGF $\beta 1$ and SIS3 significantly reduced MMP9 mRNA expression to near the levels of basal cells (Figure 5b). Furthermore, pretreatment with SIS3 alleviated the TGF $\beta 1$-induced increase in MMP9 immunoreactivity (Figure 5c). Quantification of MMP9 immunofluorescence determined that treatment with the SMAD3 inhibitor was able to significantly reduce MMP9 immunoreactivity in bEnd. 3 cells to near basal levels (Figure $5 \mathrm{~d}$ ).

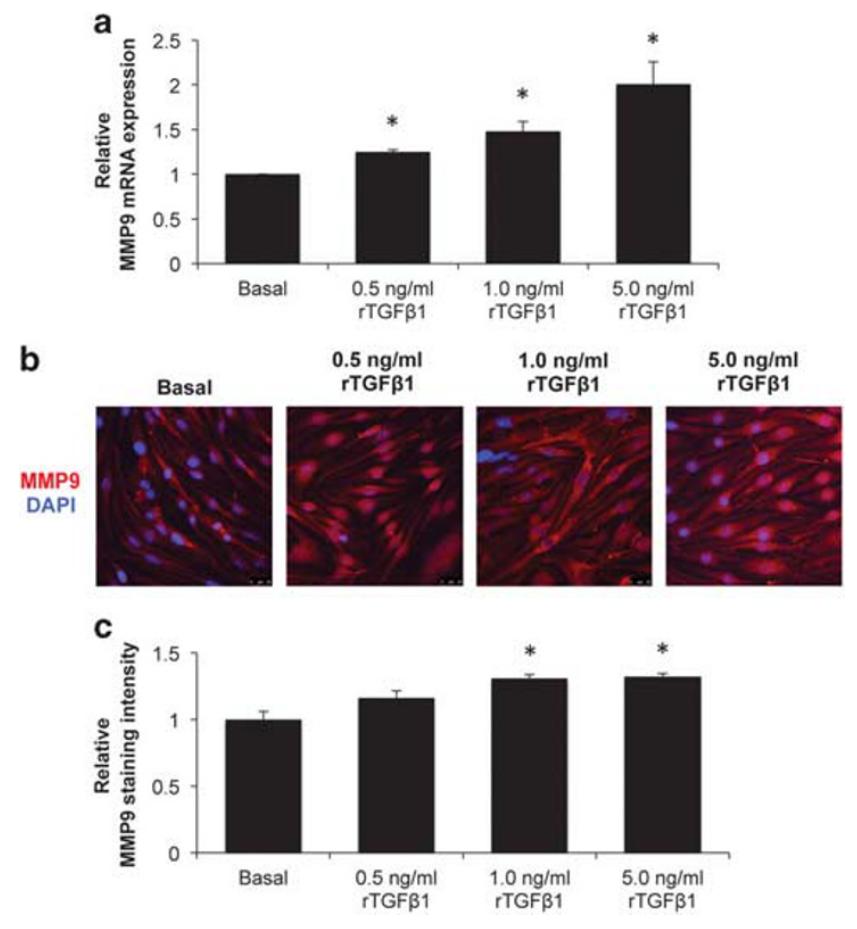

Figure 3 MMP9 is upregulated by TGF $\beta 1$ in bEnd. 3 cells. (a) MMP9 mRNA expression in bEnd. 3 cells as assessed by qPCR following treatment with rTGF $\beta$ 1. (b) Coverslips of bEnd.3 cells were stained for MMP9 (red) and DAPI (blue) as a nuclear stain following treatment with increasing doses of rTGF $\beta 1$. (c) Quantification of MMP9 immunofluorescence of bEnd. 3 coverslips following treatment with rTGF $\beta 1$. The data from mRNA and immunofluorescence quantification analyses are reported as mean \pm s.e.m. ${ }^{*}=P<0.05$ compared with basal bEnd. 3 cells.

In addition to the effects of SMAD3 antagonism on MMP9, treatment with both TGF $\beta 1$ and SIS3 was also able to rescue the downregulation of claudin-5 mRNA and protein (Figures $6 \mathrm{a}$ and b) compared with TGF $\beta 1$ treatment alone. To determine whether disruption of claudin-5 cellular localization was dependent on SMAD3, coverslips of bEnd.3 cells were treated with rTGF $\beta 1$ and SIS3. Treatment of bEnd. 3 cells with SIS3 was able to partially restore the localization of claudin-5 to the cell membrane (Figure 6c). Together, these findings demonstrate that the upregulation of MMP9 and downregulation of claudin-5 via TGF $\beta 1$ in brain endothelial cells is dependent on SMAD3.

\section{In vivo Neutralization of TGF $\beta 1$ Reduces BBB Permeability Following Liver Failure}

An Evan's blue extravasation assay was performed in mice treated with $\mathrm{AOM}$ and/or neutralizing antibodies against $\operatorname{TGF} \beta$ for $18 \mathrm{~h}$. Mice injected with AOM demonstrated a significant increase in Evan's blue dye in their brain. This increase was significantly reduced if the mice were pretreated with neutralizing antibodies against TGF $\beta$ (Figure 7a). Representative pictures of the brains of mice treated with $\mathrm{AOM}$ and/or neutralizing antibodies against $\operatorname{TGF} \beta$ support these results (Figure 7b). To demonstrate this effect more conclusively, immunohistochemistry was performed against 


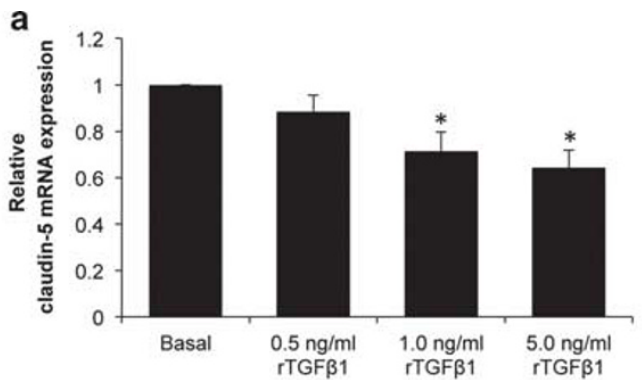

b
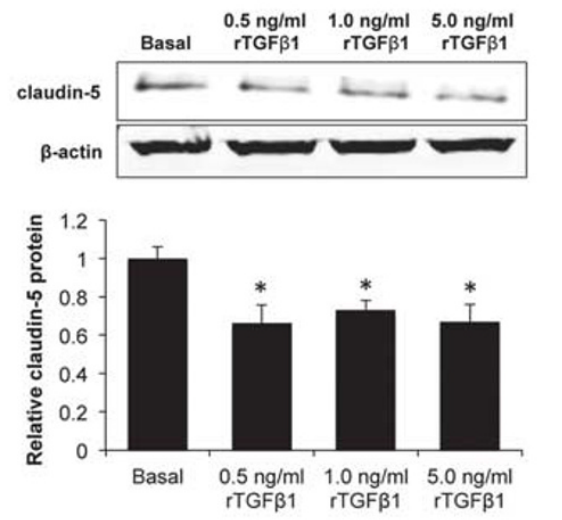

C

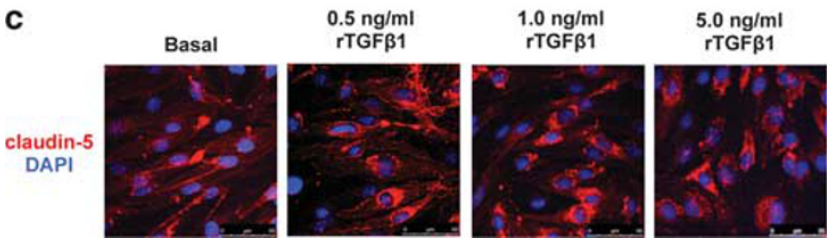

Figure 4 Claudin-5 expression is downregulated in bEnd. 3 cells by TGF $\beta 1$. (a) Claudin- 5 mRNA expression in bEnd. 3 treated with indicated doses of rTGF $\beta 1$ as assessed by qPCR. (b) Claudin- 5 representative immunoblot and quantification in bEnd.3 cells treated with indicated doses of $\mathrm{rTGF} \beta 1$. $\beta$-Actin is used as a loading control and quantifications are normalized to basal bEnd. 3 cells. (c) Claudin- 5 immunofluorescence (red) in bEnd. 3 cells treated with rTGF $\beta 1$. DAPI (blue) was used to stain nuclei. The data from mRNA and protein analyses are reported as mean \pm s.e.m. ${ }^{*}=P<0.05$ compared with basal bEnd. 3 cells.

albumin in the brains of mice treated with $\mathrm{AOM}$ and neutralizing antibodies against TGF $\beta$. This immunohistochemistry displays a significant elevation of albumin in the cortex of AOM mice, which is reduced in mice treated with neutralizing antibodies against TGF $\beta$ (Figure 7c). To visualize brain endothelial cells, immunofluorescence staining was performed for the endothelial cell marker SMI71. AOMtreated mice show discontinuous staining for SMI71, while mice pretreated with neutralizing antibodies against TGF $\beta$ show continuous staining similar to vehicle-treated mice (Figure 7d). These data support our hypothesis that inhibiting circulating TGF $\beta 1$ activity in vivo is able to restore $\mathrm{BBB}$ function following ALF.

\section{DISCUSSION}

This manuscript reports that $18 \mathrm{~h}$ following AOM injection the BBB is significantly disrupted, as measured by Evan's blue dye extravasation. Investigation into the molecular mechanisms that drive this effect determined that brain endothelial cells have increased MMP9 expression and reduced claudin-5 expression following treatment with $\mathrm{rTGF} \beta 1$. The increase in MMP9 expression and suppression of claudin-5 was found to be dependent on SMAD3 signaling, as treatment with the SMAD3 inhibitor SIS3 was able to reverse the effects of rTGF $\beta 1$ treatment. Finally, inhibition of circulating TGF $\beta 1$ in AOM-treated mice by injection of neutralizing antibodies was able to reduce albumin infiltration in the brain, reduce microvessel disruption, and significantly reduce BBB permeability compared with mice treated with AOM alone. A working model of our findings is presented in Figure 8.

This study demonstrates that the AOM model of $\mathrm{HE}$ generates a significant disruption of the $\mathrm{BBB}$ as measured by the presence of Evan's blue dye in the cerebral cortices of AOM-treated mice. Interestingly, this only occurs at later stages of $\mathrm{HE}$ when severe neurological decline is present (ataxia and minor reflex deficits are typically observed around twelve hours in this model). ${ }^{16}$ Other researchers have performed Evan's blue dye extravasation using a lower dose of $\mathrm{AOM}(50 \mathrm{mg} / \mathrm{kg})$ and have shown similar findings. ${ }^{12}$ Furthermore, rats treated with D-galactosamine to induce ALF show significant increase of Evan's blue extravasation at coma. $^{27}$ These findings mirror the more recent reports of vasogenic edema that have been reported in clinical studies in both acute and chronic liver failure. ${ }^{28,29}$ However, it has recently been proposed that $\mathrm{AOM}$ itself may be leading to a direct effect on the endothelial cells of the BBB. The monolayer experiments performed in this study indicate that AOM does not cause increased endothelial cell permeability directly, but that TGF $\beta 1$ contributes to increased monolayer permeability in this model. One research group has investigated AOM in an endothelial cell and astrocyte coculture model and found that treatment with $5 \mu \mathrm{g} / \mathrm{ml} \mathrm{AOM}$ for $24 \mathrm{~h}$ leads to increased endothelial cell permeability. ${ }^{30}$ The discrepancy in the effects of AOM on permeability may lie in the site of AOM administration. The previous study administered AOM in the media between the astrocyte and endothelial cell layer rather than directly onto the endothelial cells themselves (as would mimic the in vivo situation). The increased permeability, therefore, may be a reflection of direct toxicity of AOM on astrocytes, which may subsequently release factors that increase the endothelial cell permeability. However, these direct effects of AOM would not translate in vivo as the toxin would have to already cross the BBB to generate this effect. These conclusions about AOM not generating direct toxicity on $\mathrm{BBB}$ endothelial cells in vivo are also supported by AOM-treated mice not showing BBB permeability to near coma, as is reported in this study and by others. $^{12}$

The current study demonstrated that MMP9 expression was increased in bEnd.3 cells following treatment with $\operatorname{rTGF} \beta 1$. TGF $\beta 1$ treatment has been shown to lead to increased expression of MMP9 in corneal epithelial cells ${ }^{31}$ 
a

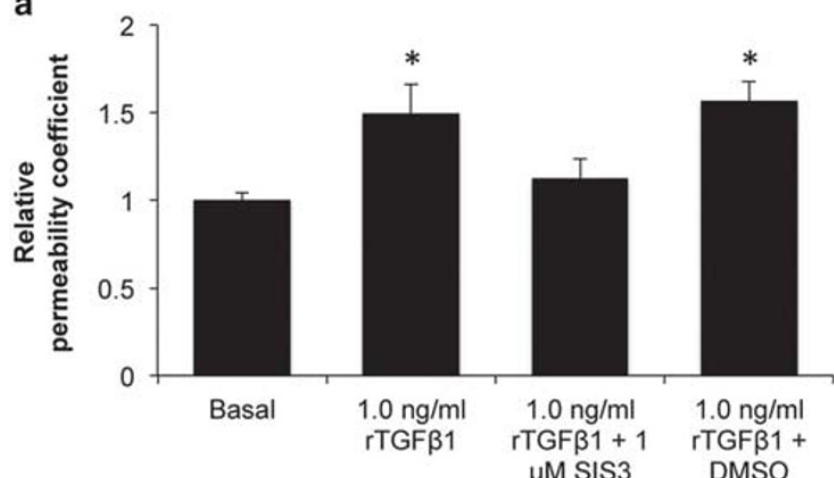

C

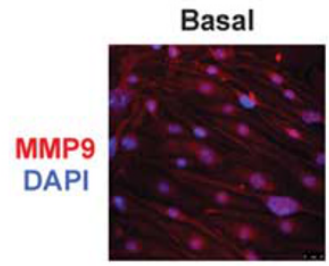

$1.0 \mathrm{ng} / \mathrm{ml} \mathrm{rTGF} \beta 1$ $+1 \mu \mathrm{M}$ SIS 3

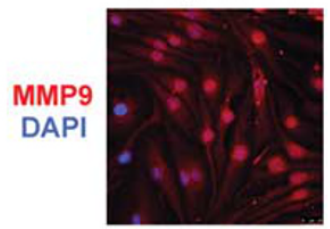

$1.0 \mathrm{ng} / \mathrm{ml}$ rTGF $\beta 1$

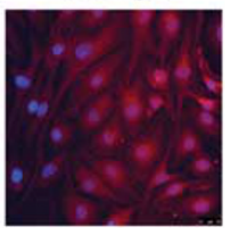

$1.0 \mathrm{ng} / \mathrm{ml}$ rTGF $\beta 1+$ DMSO

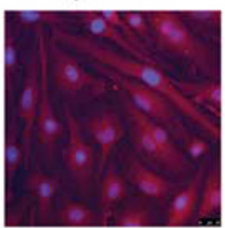

b

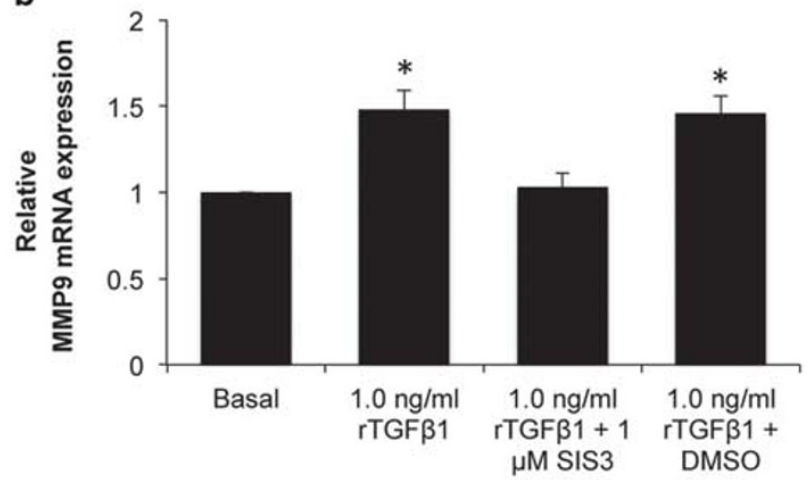

d

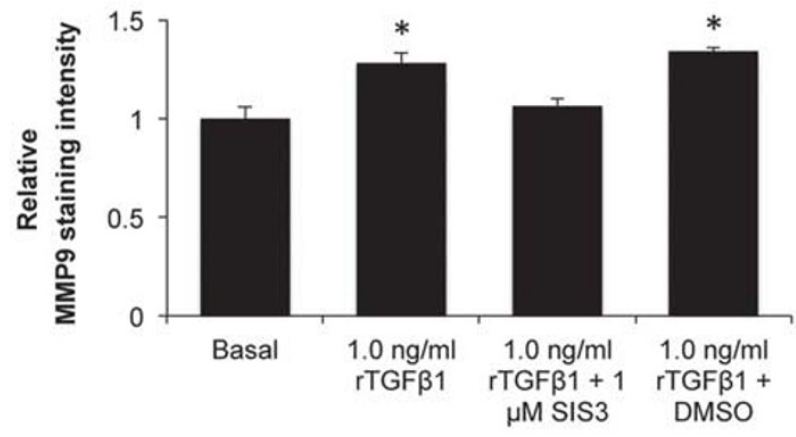

Figure 5 SMAD3 is required for upregulation of MMP9 by TGF 31 . (a) Monolayers of bEnd.3 cells were treated with rTGF $\beta 1$, the SMAD3 inhibitor SIS3, or SIS3 vehicle (DMSO) for $24 \mathrm{~h}$. Permeability was assessed by measuring diffusion of 10-kDa FITC-dextran from the top chamber to the bottom chamber and a subsequent fluorescence measurement (excitation 494/emission 520). (b) Relative MMP9 mRNA expression in bEnd.3 cells treated with rTGF $\beta 1$, the SMAD3 inhibitor SIS3, or SIS3 vehicle (DMSO) for $24 \mathrm{~h}$. (c) bEnd.3 cell coverslips were treated with rTGF $\beta 1$, the SMAD3 inhibitor SIS3, or SIS3 vehicle (DMSO) for $24 \mathrm{~h}$. Immunofluorescence against MMP9 (red) and DAPI (blue) was performed. (d) Quantification of MMP9 immunoreactivity on bEnd.3 coverslips following treatment with $\mathrm{rTGF} \beta 1$ and the SMAD3 inhibitor SIS3. The data from permeability, mRNA, and immunofluorescence quantification analyses are reported as mean \pm s.e.m. ${ }^{*}=P<0.05$ compared with basal bEnd.3 cells.

and in podocytes. ${ }^{32}$ MMP9 has many roles in dysregulation of the $\mathrm{BBB}$ as it has the capability to degrade claudin-5, occludin, zona occludens- 1 , and zona occludens $-2 .{ }^{9}$ During AOM-induced HE, the upregulation of MMP9 has been shown to disrupt tight junction expression, and pharmacological inhibition of MMP9 restores tight junction protein expression to near control levels. ${ }^{11}$ Interestingly, it has also been shown that treatment of rat brain microvascular endothelial cells with ammonia alone is able to induce MMP9 expression. ${ }^{33}$ Therefore, it is conceivable that TGF $\beta 1$ could potentially be acting in synergy with ammonia to induce MMP9 expression and further drive BBB permeability. Further studies are necessary to identify the specific cellular sources, regulation, and protein interactions of MMP9 to better understand its role in $\mathrm{BBB}$ dysfunction following liver failure.

Previous research into the effects of ALF on tight junction protein dysregulation has been conflicting. One group of researchers found that AOM-treated mice have disruptions of occludin, claudin- 5 , zona occludens- 1 , and zona occludens- 2 protein expression as assessed with western blots. ${ }^{11}$ Conversely, other researchers have used the AOM model and observed no changes in immunoblot tight junction protein expression. ${ }^{34}$ Our studies focused only on the effects of TGF $\beta 1$ on claudin-5 expression and demonstrated that treatment of bEnd.3 cells with $\operatorname{rTGF} \beta 1$ downregulated claudin-5 and led to the translocation of this protein from the cell membrane to the cytosol. Claudin-5 downregulation has been shown to lead to increased BBB permeability following hypoxia, ${ }^{35}$ focal cerebral cooling, ${ }^{36}$ and ischemia reperfusion injury. ${ }^{37}$ Also, other studies have found that TGF $\beta 1$ can lead to the direct downregulation of claudin-5. ${ }^{38}$ Thus, these findings support the concept that circulating TGF $\beta 1$ promotes the $\mathrm{BBB}$ permeability observed in vivo primarily through the downregulation of claudin-5. To our knowledge, this is the first study to show changes in both claudin-5 and MMP9 in bEnd.3 cells following treatment with $\operatorname{rTGF} \beta 1$. However, whether the effects of TGF $\beta 1$ on 
claudin-5 and MMP-9 are part of the same pathway or are the result of two separate TGF $\beta 1$-mediated processes is unknown. Regardless, the overall outcome is an increase in BBB permeability as a result of TGF $\beta 1$ signaling.

TGF $\beta 1$ transduces its signal via activation of its receptor and subsequent phosphorylation of the SMAD proteins, $\mathrm{SMAD} 2$ or SMAD $3 .{ }^{39}$ We determined that SMAD3 was generating the majority of the effects that we were seeing both

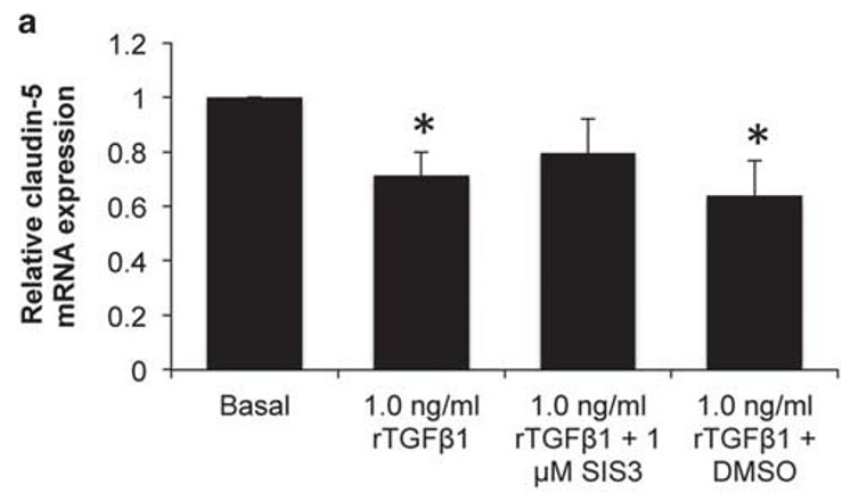

b
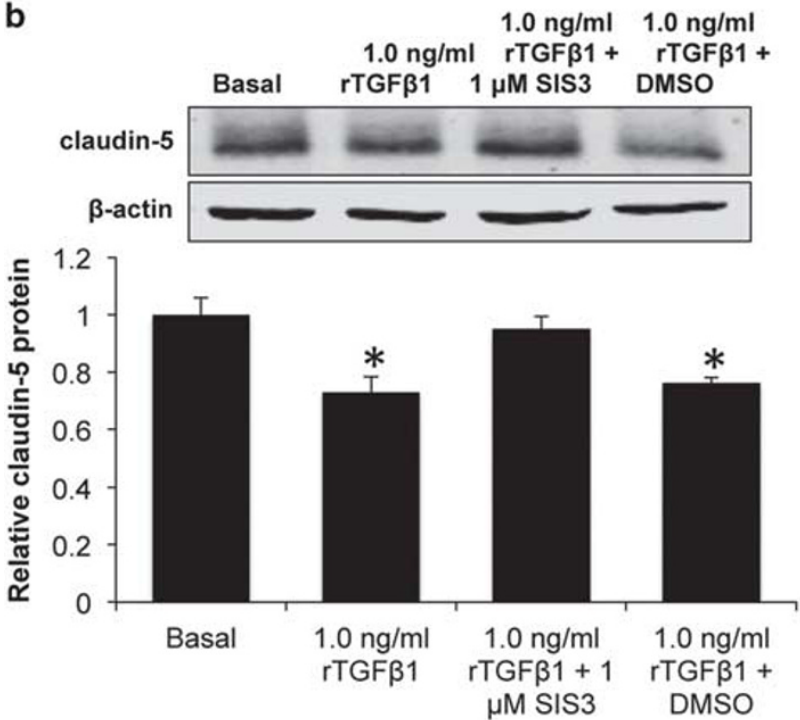

c
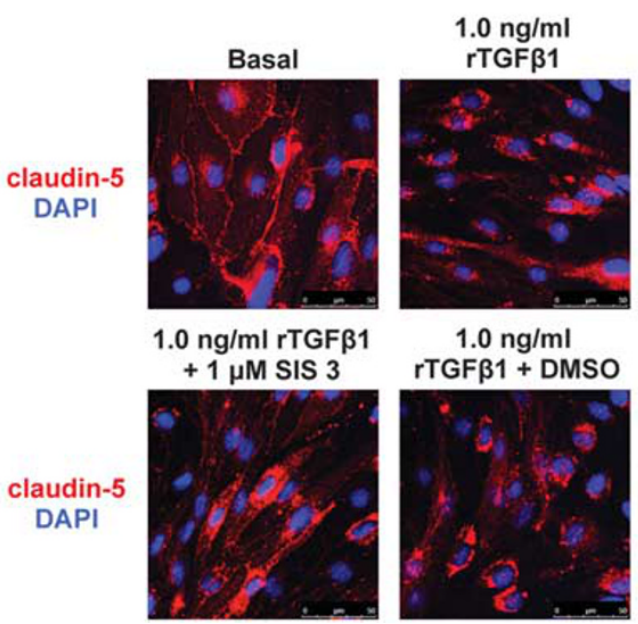

in vivo and in vitro by showing that treatment with SIS3 was able to reverse the effects of TGF $\beta 1$. Interestingly, other studies have inhibited $\operatorname{TGF} \beta$ receptor kinase activity, which reduced SMAD3 activity, and observed elevated claudin-5 expression. ${ }^{40}$ In addition, human meningeal cells treated with a SMAD3 inhibitor were able to attenuate TGF $\beta 1$-dependent MMP9 upregulation. ${ }^{41}$ Thus, there is strong evidence supporting dysregulation of both claudin-5 and MMP9 by a

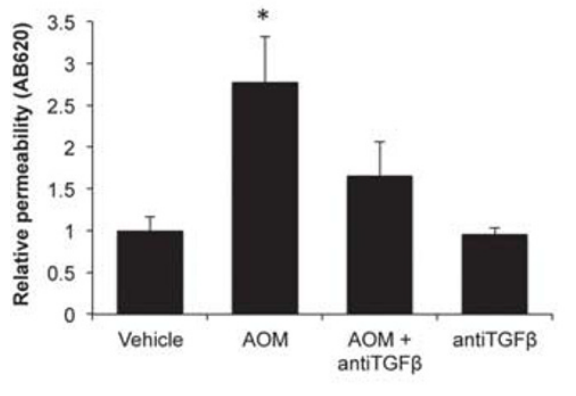

b

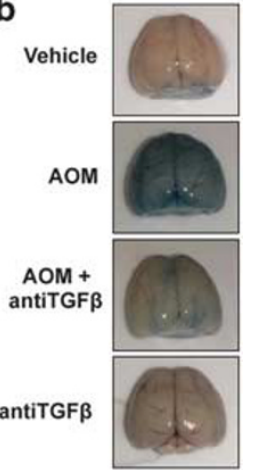

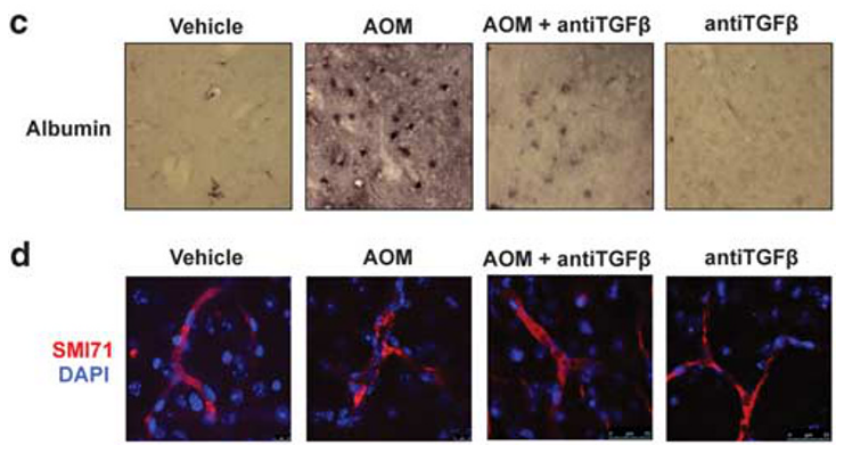

Figure 7 Treatment of AOM mice with neutralizing antibodies against TGF $\beta$ reduces BBB dysfunction. (a) Evan's blue dye permeability assay of mice treated with AOM or with neutralizing antibodies against TGF $\beta$ for $18 \mathrm{~h}(n=4)$. Permeability was measured by measuring absorbance of Evan's blue dye $(620 \mathrm{nM})$. (b) Representative images of Evan's blue dye extravasation in vehicle, AOM, AOM+antiTGF $\beta$, and antiTGF $\beta$ mice. (c) Immunohistochemistry against albumin in the cortex of vehicle, AOM, AOM+antiTGF $\beta$, and antiTGF $\beta$ mice. (d) Immunofluorescence staining for the endothelial cell marker SMI71 (red) with DAPI (blue) used as a nuclear stain in the cortex of vehicle, AOM, AOM+antiTGF $\beta$, and antiTGF $\beta$ mice. Data in the permeability assay are reported as mean \pm s.e.m. ${ }^{*}=P<0.05$ compared with vehicle-treated mice.

Figure 6 Claudin- 5 downregulation via TGF $\beta 1$ occurs through a SMAD3dependent mechanism. (a) Claudin-5 mRNA expression in bEnd.3 cells treated with $\mathrm{rTGF} \beta 1$, the SMAD3 inhibitor SIS3, or SIS3 vehicle (DMSO) for $24 \mathrm{~h}$. (b) Claudin-5 protein in bEnd. 3 cells treated with rTGF $\beta 1$, the SMAD3 inhibitor SIS3, or SIS3 vehicle (DMSO) for $24 \mathrm{~h}$ as assessed by immunoblotting. $\beta$-Actin is used as a loading control and quantifications are normalized to basal bEnd.3 cells. (c) Claudin-5 (red)

immunofluorescence in bEnd. 3 cells treated with $\operatorname{rTGF} \beta 1$, the SMAD3 inhibitor SIS3, or SIS3 vehicle (DMSO) for $24 \mathrm{~h}$. DAPI (blue) was used as a nuclear stain. The data from mRNA and immunoblot analyses are reported as mean \pm s.e.m. ${ }^{*}=P<0.05$ compared with basal bEnd. 3 cells. 


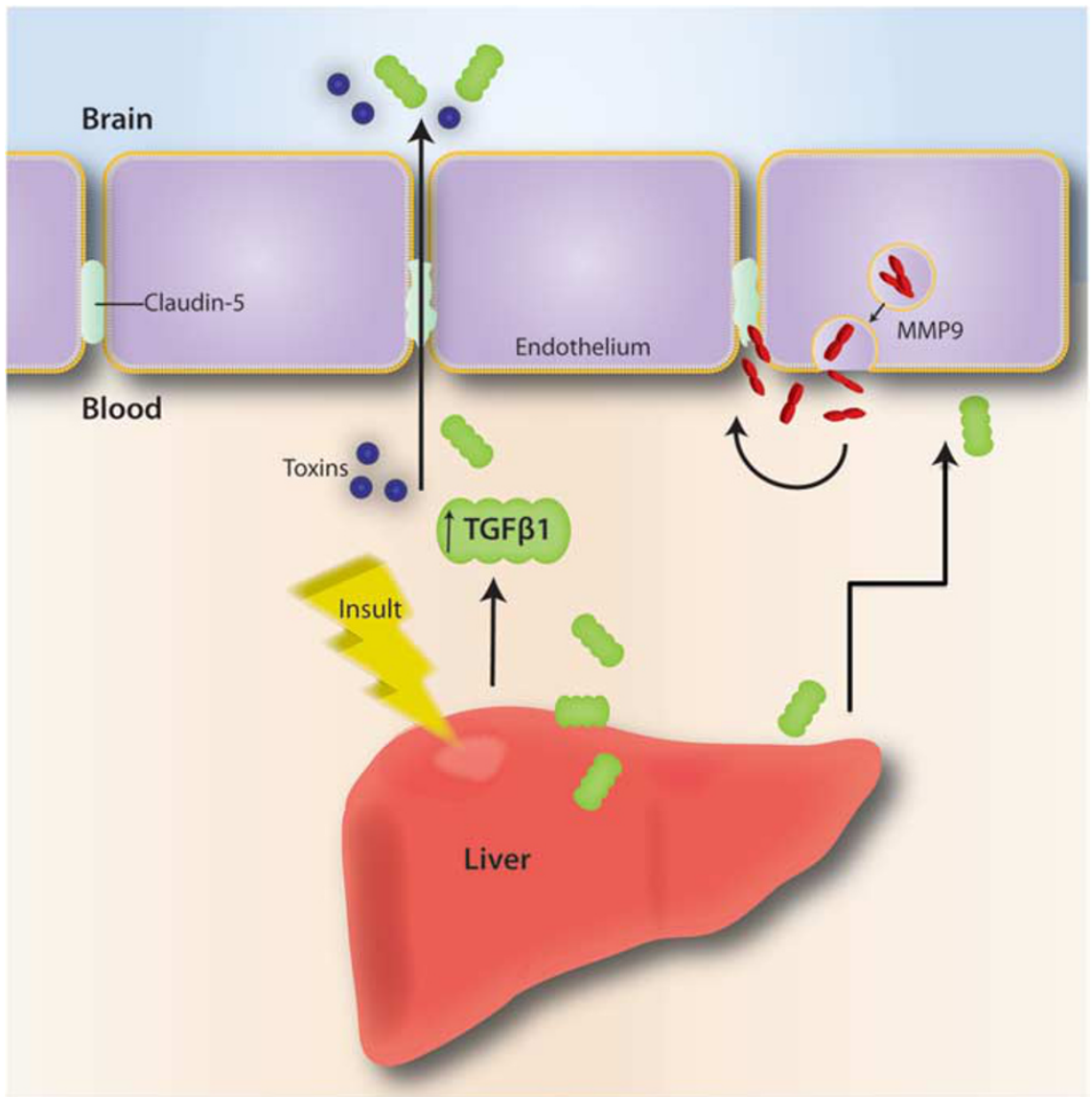

Figure 8 Working model of TGF $\beta$-induced permeability of BBB. Following liver insult, TGF $\beta 1$ is released from the liver into the circulation. This generates two subsequent effects. One is the TGF $\beta 1$-dependent upregulation of MMP9 in endothelial cells with subsequent release into the circulation and digestion of claudin-5. The other effect is a downregulation of claudin-5 via TGF $\beta 1$ and a subsequent disruption of tight junctions. Together these effects allow signaling proteins like TGF $\beta 1$ and toxins to pass through the BBB and enter into the brain to exacerbate HE pathology.

TGF $\beta 1$ via a SMAD3-dependent mechanism. As this was the first study manipulating TGF $\beta 1 /$ SMAD3 signaling in these cells, it is possible that this signaling pathway could be generating other effects on these cells that have not been investigated, such as affecting junctional adhesion molecules or proteins of the adherens junction.

Sequestration of circulating TGF $\beta 1$ in vivo demonstrated a significant role for TGF $\beta 1$ in promoting permeability of the $\mathrm{BBB}$ in this model of ALF. One downstream consequence of TGF $\beta 1$ is the activation of phosphatidylinositol-3 kinase/Akt pathway signaling ${ }^{42}$ which has been demonstrated to promote vascular permeability in cancer models. ${ }^{43,44}$ Also, this pathway has been shown to induce BBB permeability following focal cerebral ischemia, ${ }^{45} \mathrm{HIV}$-induced $\mathrm{BBB}$ disruption, ${ }^{46}$ and traumatic brain injury. ${ }^{47}$ In addition to this, we have previously identified that inhibition of TGF $\beta 1$ can lead to increased expression of the hedgehog transcription factor Gli1. ${ }^{16}$ Indeed, hedgehog pathway activation has been shown to be protective in experimental autoimmune encephalomyelitis, a mouse model of multiple sclerosis, by promoting blood-brain barrier integrity. ${ }^{48}$ Also, treatment of mice with polydatin, which elevates Gli1, has been shown to restore $\mathrm{BBB}$ function following ischemic insult. ${ }^{49}$ These findings suggest that inhibiting TGF $\beta 1$ could promote $\mathrm{BBB}$ integrity via suppression of vascular permeability pathway signaling as well as by inducing factors to promote BBB vascular integrity. Further research into the specific signaling pathways in brain endothelial cells that are dysregulated by $\operatorname{TGF} \beta 1$ are still warranted and are currently ongoing in the laboratory.

Together, our findings support that TGF $\beta 1$ is driving BBB permeability via downregulation of claudin-5 and upregulation of MMP9 and that these effects are dependent on SMAD3. These results support that manipulations of TGF $\beta 1$ or therapies to target SMAD3 may be potential therapeutic targets to treat ALF patients who have the potential to develop HE.

\section{ACKNOWLEDGMENTS}

We thank Dinorah Carrillo for technical assistance on this project. This study was funded by an NIH R01 award (DK082435), an NIH K01 award (DK078532) and a Scott \& White Intramural grant award (No: 050339) to Dr. DeMorrow. 
This material is the result of work supported with resources and the use of facilities at the Central Texas Veterans Health Care System, Temple, Texas.

\section{DISCLOSURE/CONFLICT OF INTEREST}

The authors declare no conflict of interest.

1. Rolando N, Wade J, Davalos $\mathrm{M}$ et al. The systemic inflammatory response syndrome in acute liver failure. Hepatology 2000;32:734-739.

2. Walsh TS, Wigmore SJ, Hopton $\mathrm{P}$ et al. Energy expenditure in acetaminophen-induced fulminant hepatic failure. Crit Care Med 2000;28:649-654.

3. Bernal W, Auzinger G, Dhawan A et al. Acute liver failure. Lancet 2010;376:190-201.

4. Rama Rao KV, Norenberg MD. Brain energy metabolism and mitochondrial dysfunction in acute and chronic hepatic encephalopathy. Neurochem Int 2012;60:697-706.

5. Hazell AS, Butterworth RF. Hepatic encephalopathy: An update of pathophysiologic mechanisms. Proc Soc Exp Biol Med 1999;222: 99-112.

6. Lockwood AH, Yap EW, Wong WH. Cerebral ammonia metabolism in patients with severe liver disease and minimal hepatic encephalopathy. J Cereb Blood Flow Metab 1991;11:337-341.

7. Ballabh P, Braun A, Nedergaard M. The blood-brain barrier: an overview: structure, regulation, and clinical implications. Neurobiol Dis 2004;16:1-13.

8. Hawkins BT, Davis TP. The blood-brain barrier/neurovascular unit in health and disease. Pharmacol Rev 2005;57:173-185.

9. Yang Y, Estrada EY, Thompson JF et al. Matrix metalloproteinasemediated disruption of tight junction proteins in cerebral vessels is reversed by synthetic matrix metalloproteinase inhibitor in focal ischemia in rat. J Cereb Blood Flow Metab 2007;27:697-709.

10. Shimojima N, Eckman CB, McKinney $M$ et al. Altered expression of zonula occludens-2 precedes increased blood-brain barrier permeability in a murine model of fulminant hepatic failure. J Invest Surg 2008;21:101-108.

11. Chen F, Ohashi N, Li W et al. Disruptions of occludin and claudin-5 in brain endothelial cells in vitro and in brains of mice with acute liver failure. Hepatology 2009;50:1914-1923.

12. Nguyen $\mathbf{J H}$, Yamamoto $\mathrm{S}$, Steers J et al. Matrix metalloproteinase-9 contributes to brain extravasation and edema in fulminant hepatic failure mice. J Hepatol 2006;44:1105-1114.

13. Yoshimura A, Wakabayashi Y, Mori T. Cellular and molecular basis for the regulation of inflammation by TGF-beta. J Biochem 2010;147: 781-792.

14. Shi Y, Massague J. Mechanisms of TGF-beta signaling from cell membrane to the nucleus. Cell 2003;113:685-700.

15. Eguchi S, Kamlot A, Ljubimova J et al. Fulminant hepatic failure in rats: survival and effect on blood chemistry and liver regeneration. Hepatology 1996;24:1452-1459.

16. McMillin M, Galindo C, Pae HY et al. Gli1 activation and protection against hepatic encephalopathy is suppressed by circulating transforming growth factor $\beta 1$ in mice. J Hepatology 2014;61: 1260-1266.

17. Goldberg PL, MacNaughton DE, Clements RT et al. p38 MAPK activation by TGF-beta1 increases MLC phosphorylation and endothelial monolayer permeability. Am J Physiol Lung Cell Mol Physiol 2002;282:L146-L154.

18. Behzadian MA, Wang XL, Windsor LJ et al. TGF-beta increases retinal endothelial cell permeability by increasing MMP-9: possible role of glial cells in endothelial barrier function. Invest Opthalmol Vis Sci 2001;42:853-859.

19. McMillin M, Frampton G, Thompson $M$ et al. Neuronal CCL2 is upregulated during hepatic encephalopathy and contributes to microglia activation and neurological decline. J Neuroinflamm 2014; 11:121.

20. Manaenko A, Chen H, Kammer J et al. Comparison Evans Blue injection routes: Intravenous versus intraperitoneal, for measurement of bloodbrain barrier in a mice hemorrhage model. J Neurosci Methods 2011;195:206-210.

21. Quinn M, McMillin M, Galindo C et al. Bile acids permeabilize the blood brain barrier after bile duct ligation in rats via Rac1-dependent mechanisms. Dig Liver Dis 2014;46:527-534.
22. Yuan SY, Rigor RR. Methods for measuring permeabilityRegulation of Endothelial Barrier Function. Morgan \& Claypool Life Sciences: San Rafael (CA), 2010.

23. DeMorrow S, Francis H, Gaudio $\mathrm{E}$ et al. The endocannabinoid anandamide inhibits cholangiocarcinoma growth via activation of the noncanonical Wnt signaling pathway. Am J Physiol Gastrointest Liver Physiol 2008;295:G1150-G1158.

24. Livak KJ, Schmittgen TD. Analysis of relative gene expression data using real-time quantitative PCR and the 2(-Delta Delta C(T)) Method. Methods 2001;25:402-408.

25. Khan SA, Joyce J, Tsuda T. Quantification of active and total transforming growth factor-beta levels in serum and solid organ tissues by bioassay. BMC Res Notes 2012;5:636.

26. Heldin $\mathrm{CH}$, Miyazono $\mathrm{K}$, ten Dijke P. TGF-beta signalling from cell membrane to nucleus through SMAD proteins. Nature 1997;390: 465-471.

27. Yamamoto S, Nguyen JH. TIMP-1/MMP-9 imbalance in brain edema in rats with fulminant hepatic failure. J Surg Res 2006;134:307-314.

28. Rai V, Nath K, Saraswat VA et al. Measurement of cytotoxic and interstitial components of cerebral edema in acute hepatic failure by diffusion tensor imaging. J Magn Reson Imaging 2008;28:334-341.

29. Kale RA, Gupta RK, Saraswat VA et al. Demonstration of interstitial cerebral edema with diffusion tensor MR imaging in type $C$ hepatic encephalopathy. Hepatology 2006;43:698-706.

30. Jayakumar AR, Ruiz-Cordero R, Tong XY et al. Brain edema in acute liver failure: role of neurosteroids. Arch Biochem Biophys 2013;536: $171-175$.

31. Kim HS, Luo L, Pflugfelder SC et al. Doxycycline inhibits TGF-beta1induced MMP-9 via Smad and MAPK pathways in human corneal epithelial cells. Invest Ophthalmol Vis Sci 2005;46:840-848.

32. Huang HC, Liu SY, Liang $Y$ et al. Transforming growth factor-beta1 stimulates matrix metalloproteinase-9 production through ERK activation pathway and upregulation of Ets-1 protein. Zhonghua yi xue za zhi 2005;85:328-331.

33. Skowronska $M$, Zielinska $M$, Wojcik-Stanaszek $L$ et al. Ammonia increases paracellular permeability of rat brain endothelial cells by a mechanism encompassing oxidative/nitrosative stress and activation of matrix metalloproteinases. J Neurochem 2012;121:125-134.

34. Bémeur $C$, Chastre $A$, Desjardins $P$ et al. No changes in expression of tight junction proteins or blood-brain barrier permeability in azoxymethane-induced experimental acute liver failure. Neurochem Int 2010;56:205-207.

35. Koto T, Takubo K, Ishida S et al. Hypoxia disrupts the barrier function of neural blood vessels through changes in the expression of claudin-5 in endothelial cells. Am J Pathol 2007;170:1389-1397.

36. Inamura $A$, Adachi $\mathrm{Y}$, Inoue $\mathrm{T}$ et al. Cooling treatment transiently increases the permeability of brain capillary endothelial cells through translocation of claudin-5. Neurochem Res 2013;38:1641-1647.

37. Jiao $H$, Wang $Z$, Liu $Y$ et al. Specific role of tight junction proteins claudin-5, occludin, and ZO-1 of the blood-brain barrier in a focal cerebral ischemic insult. J Mol Neurosci 2011;44:130-139.

38. Ronaldson PT, Demarco KM, Sanchez-Covarrubias L et al. Transforming growth factor-beta signaling alters substrate permeability and tight junction protein expression at the blood-brain barrier during inflammatory pain. J Cereb Blood Flow Metab 2009;29:1084-1098.

39. Larsson J, Karlsson S. The role of Smad signaling in hematopoiesis. Oncogene 2005;24:5676-5692.

40. Watabe T, Nishihara A, Mishima $\mathrm{K}$ et al. TGF-beta receptor kinase inhibitor enhances growth and integrity of embryonic stem cellderived endothelial cells. J Cell Biol 2003;163:1303-1311.

41. Okamoto T, Takahashi S, Nakamura E et al. Transforming growth factor-beta1 induces matrix metalloproteinase- 9 expression in human meningeal cells via ERK and Smad pathways. Biochem Biophys Res Commun 2009;383:475-479.

42. Kato M, Putta S, Wang M et al. TGF-beta activates Akt kinase through a microRNA-dependent amplifying circuit targeting PTEN. Nat Cell Biol 2009;11:881-889.

43. Hu L, Hofmann J, Jaffe RB. Phosphatidylinositol 3-kinase mediates angiogenesis and vascular permeability associated with ovarian carcinoma. Clin Cancer Res 2005;11:8208-8212.

44. Yuan TL, Choi HS, Matsui A et al. Class $1 \mathrm{~A}$ PI3K regulates vessel integrity during development and tumorigenesis. Proc Natl Acad Sci USA 2008;105:9739-9744. 
45. Kilic E, Kilic U, Wang $Y$ et al. The phosphatidylinositol-3 kinase/Akt pathway mediates VEGF's neuroprotective activity and induces blood brain barrier permeability after focal cerebral ischemia. FASEB J 2006;20:1185-1187.

46. Yang B, Singh S, Bressani R et al. Cross-talk between STAT1 and PI3K AKT signaling in HIV-1-induced blood-brain barrier dysfunction: role of CCR5 and implications for viral neuropathogenesis. J Neurosci Res 2010;88:3090-3101.

47. Tchantchou F, Zhang Y. Selective inhibition of alpha/beta-hydrolase domain 6 attenuates neurodegeneration, alleviates blood brain barrier breakdown, and improves functional recovery in a mouse model of traumatic brain injury. J Neurotrauma 2013;30:565-579.

48. Alvarez Jl, Dodelet-Devillers A, Kebir $\mathrm{H}$ et al. The Hedgehog pathway promotes blood-brain barrier integrity and CNS immune quiescence. Science 2011:334:1727-1731.

49. Ji $\mathrm{H}$, Zhang $\mathrm{X}, \mathrm{Du} \mathrm{Y}$ et al. Polydatin modulates inflammation by decreasing NF-kappaB activation and oxidative stress by increasing Gli1, Ptch1, SOD1 expression and ameliorates blood-brain barrier permeability for its neuroprotective effect in PMCAO rat brain. Brain Res Bull 2012;87:50-59. 